\title{
Galaxy dynamics and supermassive black holes in the era of large telescope
}

\section{Laura Ferrarese}

Herzberg Institute of Astrophysics, Canada

\begin{abstract}
Dynamical studies of the centers of galaxies have received a tremendous push forward with the launch of the Hubble Space Telescope. Thanks to its superb resolution power, HST has made it possible not only to convincingly demonstrate the existence of supermassive black holes (SBHs) in galactic nuclei, but also to investigate how SBHs relate to the overall structure of the host galaxy. In spite of this, many questions remain unanswered, for instance, how do scaling relations for SBHs evolve with cosmic time? What is the exact characterization of the local SBH mass function? Are there upper and lower limits to the mass a SBH can attain? The next generation of $30 \mathrm{~m}$ telescopes will provide the leap in resolution capabilities and collective power which is necessary to address the above questions. I will discuss some of the main science drivers in the field of galaxy dynamics, and the instrument requirements needed to achieve them.
\end{abstract}

